\title{
INSUFICIENCIA RENAL AGUDA POSTRENAL
}

\author{
OLIGURIA DEBIDA A OBSTRUCCION DE LAS VIAS URINARIAS
}

J. D. ORDOÑEZ, H. TORRES, R. D'ACHIARDI

Se describen cuatro casos ilustrativos de oliguria aguda producida por obstrucción urinaria y se discute el diagnóstico diferencial de esta entidad con las demás causas de oliguria y de insuficiencia renal aguda. Se incluye también una breve discusión de la patofisiología de los trastornos funcionales renales producidos por la elevación de la presión hidrostática dentro de las vías urinarias y de su manejo clínico.

La disminución del volumen urinario con azotemia creciente es un hallazgo clínico frecuente que requiere del médico un diagnóstico diferencial preciso y una terapia rápida. El problema consiste en diferenciar los casos de insuficiencia renal aguda, que implican daño parenquimatoso del órgano y requieren el uso de hemodiálisis en un centro especializado, de las

Dr. Juan Daniel Ordóñez O.: Jefe, Sección de Nefrología; Dr. Hernán Torres I.: Jefe. Departamento de Medicina y Dr. Roberto D'Achiardi R.: Nefrólogo Asociado. Hospital Militar de Bogotá.

Solicitud de separatas al Dr. Ordóñez. causas extrarrenales de oliguria, rápidamente reversibles y más fáciles de tratar.

Las causas de oliguria se clasifican generalmente en prerrenales, renales y postrenales. La llamada insuficiencia prerrenal (Tabla 1) se debe a una disminución del

Tabla 1- Causas de oliguria prerrenal.

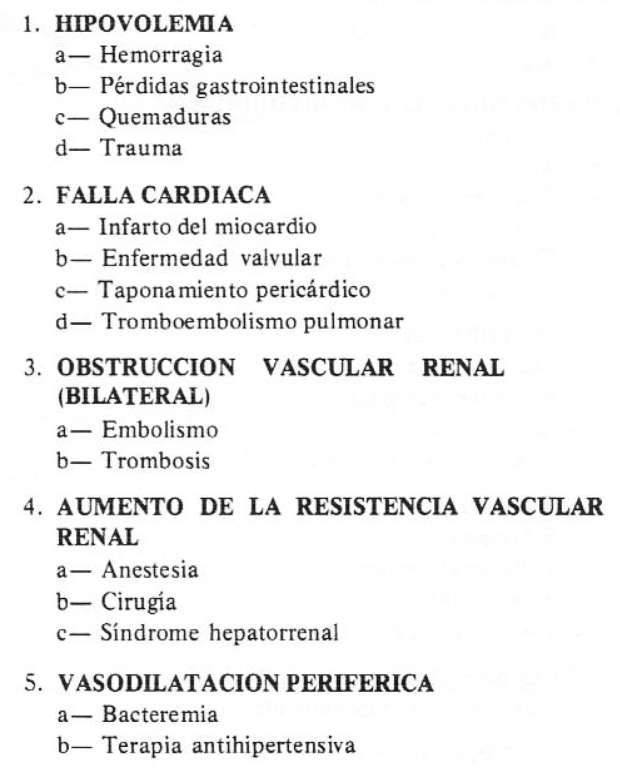


flujo plasmático del órgano, que puede ocurrir en casos de pérdida de volumen extracelular de cualquier origen; de obstrucción vascular renal o de insuficiencia cardiaca (1-3). El diagnóstico es relativamente fácil porque las entidades clínicas que la producen son en su mayoría evidentes al examen; además, el estudio de la orina generalmente muestra un bajo contenido de sodio (menos de $30 \mathrm{meq} / \mathrm{L}$ ), una osmolaridad elevada y un sedimento normal $(4,5)$. Ninguno de estos hallazgos sería posible sin el normal funcionamiento de los túbulos renales, y por esto descartan su daño estructural.

La insuficiencia renal aguda de tipo parenquimatoso implica el daño estructural y funcional de un número considerable de nefrones; el contenido urinario de sodio es alto (generalmente de más de $30 \mathrm{meq} / \mathrm{L}$ ), la osmolaridad urinaria baja y el sedimento urinario generalmente anormal $(4,5)$. La Tabla 2 ilustra algunas de las situaciones clínicas en las cuales se presenta esta entidad.

Tabla 2-Causas de insuficiencia renal aguda.

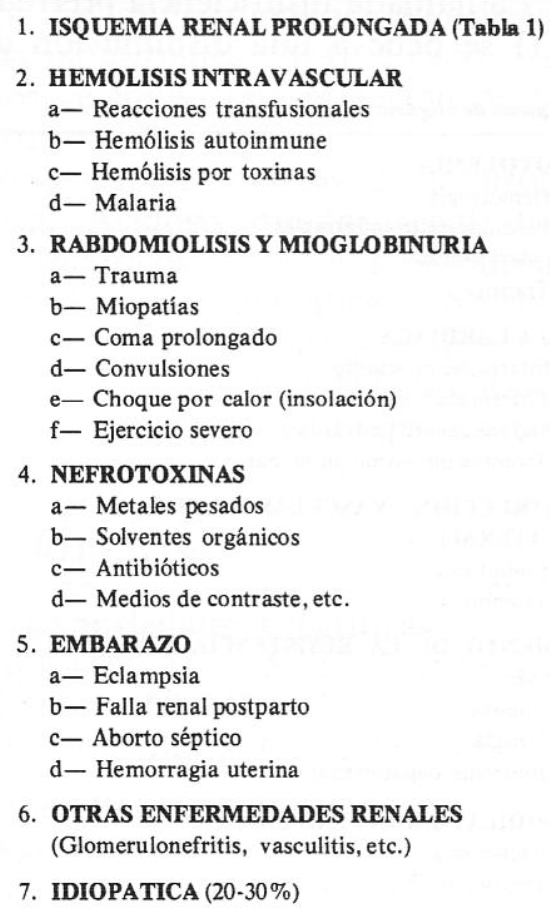

La obstrucción de las vías urinarias es causa mucho más rara de oliguria y la literatura médica al respecto es escasa. Los casos descritos a continuación, referidos en un corto plazo a nuestra institución, ilustran el problema:

\section{Caso No. 1}

G.R., un hombre blanco de 59 años de edad, consultó al Hospital por oliguria progresiva hasta anuria completa durante la semana anterior al ingreso; existían antecedentes de dificultad para iniciar la micción y polaquiuria durante 5 años, que se habían agudizado 20 días antes del ingreso. No había historia de enfermedad renal; el examen reveló un paciente álgido, afebril, quejándose de dolor abdominal. El pulso era de 100/min., la T.A.de 140/80; los pulmones eran claros, los ruidos cardiacos normales, el abdomen blando, moderadamente doloroso a la palpación en hipogastrio, sin globo vesical definido. El tacto rectal reveló una próstata aumentada de tamaño, de unos 60 grs. y de características benignas. El electrocardiograma mostró ondas T elevadas y complejos QRS de duración prolongada. El hematocrito era $37 \%$, el recuento leucocitario $20.700 / \mathrm{mm}^{3}$, con $89 \%$ polisegmentados, $3 \%$ cayados $8 \%$ linfocitos; la creatinina plasmática $10.8 \mathrm{mg} \%$, el nitrógeno ureico $81.3 \mathrm{mg}$, el sodio $143 \mathrm{meq} / \mathrm{L}$, el potasio $8.6 \mathrm{meq} / \mathrm{L}$, los cloruros $111 \mathrm{meq} / \mathrm{L}$; el examen del sedimento urinario mostró abundantes bacterias y piocitos. Se colocó una sonda de Foley y se obtuvieron más de 3.000 c.c. en colecciones sucesivas durante quince minutos; la orina era de apariencia turbia y el cultivo produjo más de 100.000 colonias de E. Coli. Se inició tratamiento con Trimetoprim-Sulfa y soluciones parenterales de cloruro y bicarbonato de sodio; en pocas horas el potasio bajó a $6.5 \mathrm{meq} / \mathrm{L}$ y el trazado electrocardiográfico se normalizó. Durante los días siguientes la diuresis fluctuó alrededor de 3 litros diarios y el paciente presentó hipotensión ortostática, deshidratación moderada e hipokalemia, que hicieron necesario un cuidadoso manejo hidroelectrolítico (Gráfica 1). La creatinina plasmática bajó progresivamente hasta estabilizarse alrededor de $4 \mathrm{mg}$. \%; el paciente fue sometido a una prostatectomía transvesical y dado de alta en el noveno día postoperatorio sin otros cambios detectables en la función renal.

\section{Caso No. 2}

B.B., una mujer blanca de 38 años de edad, consultó al Hospital por oliguria progresiva hasta anuria total de 10 días de evolución, disnea y ortopnea durante los 4 días anteriores al ingreso. No había historia de enfermedad renal previa pero existía el antecedente de hipertensión arterial irregularmente tratada durante el año anterior al ingreso. El examen físico reveló disnea, ortopnea, distensión yugular hasta los ángulos mandibulares, taquicardia de 
$104 /$ min. con galope, hepatomegalia y edema sacro y de miembros inferiores. No había masas abdominales palpables. El hematocrito era $40 \%$, el recuento leucocitario $45.200 / \mathrm{mm}^{3}$ con $92 \%$ polimorfonucleares y $6 \%$ cayados; el nitrógeno ureico $120 \mathrm{mg} . \%$, la creatinina $13.6 \%$, el sodio $139 \mathrm{meq} / \mathrm{L}$., el potasio $7.9 \mathrm{meq} / \mathrm{L}$. y el bicarbonato $18 \mathrm{meq} / \mathrm{L}$. La radiografia de tórax mostró cardiomegalia y congestión pulmonar. Una cistoscopía reveló gran edema ampolloso del meato ureteral derecho; el meato ureteral izquierdo no se pudo visualizar. Una pielografía retrógrada reveló obstrucción entre los tercios superior y medio del ureter derecho, que no pudo ser superada con el catéter. La paciente se dializó una vez y se inició terapia con Gentamicina. La exploración quirúrgica al día siguiente mostró un cálculo enclavado en la posición indicada por la pielografía y una masa sobre el polo renal inferior que resultó ser un carcinoma renal al estudio histopatológico; el riñon izquierdo no se encontró y se consideró aplásico. Se practicó una nefrostomía derecha. Durante los primeros días postoperatorios la paciente presentó diuresis abundante que no se pudo medir con exactitud, pero que fue superior a los 2 litros diarios. El potasio sérico bajó a $3 \mathrm{meq} / \mathrm{L}$. y se hizo necesario un cuidadoso balance hidroelectrolítico. En el octavo día postoperatorio la paciente fue dada de alta con una creatinina plasmática de $1.6 \mathrm{mg}$. \% y con electrolitos y leucograma normales.

\section{Caso No. 3}

G.O., una mujer blanca de 58 años de edad, fue referida al servicio de nefrología por su médico particular debido a marcada retención nitrogenada y oliguria progresiva e irregular durante varios días. Dos meses antes del ingreso la paciente notó el crecimiento acelerado de una masa pélvica anteriormente diagnosticada como un mioma uterino;una semana antes del ingreso presentó disminución del volumen urinario y vómito persistente. El día del ingreso al hospital la paciente permaneció completamente anúrica; el examen físico reveló hidratación normal, pulmones y corazón normales, pulso de $80 /$ min., T.A. $140 / 80$ y una masa pélvica firme, moderadamente dolorosa, que se extendía hasta 12 $\mathrm{cm}$. por encima del pubis y hacía cuerpo con el útero. La creatinina plasmática era de $10.7 \mathrm{mg}$. \%, el nitrógeno ureico $86.6 \mathrm{mg}$. \%, el sodio $136 \mathrm{meq} / \mathrm{L}$., el potasio $6.2 \mathrm{meq} / \mathrm{L}$, el hematocrito $42 \%$ y el recuento leucocitario de $13.000 / \mathrm{mm}^{3}$ con discreta desviación a

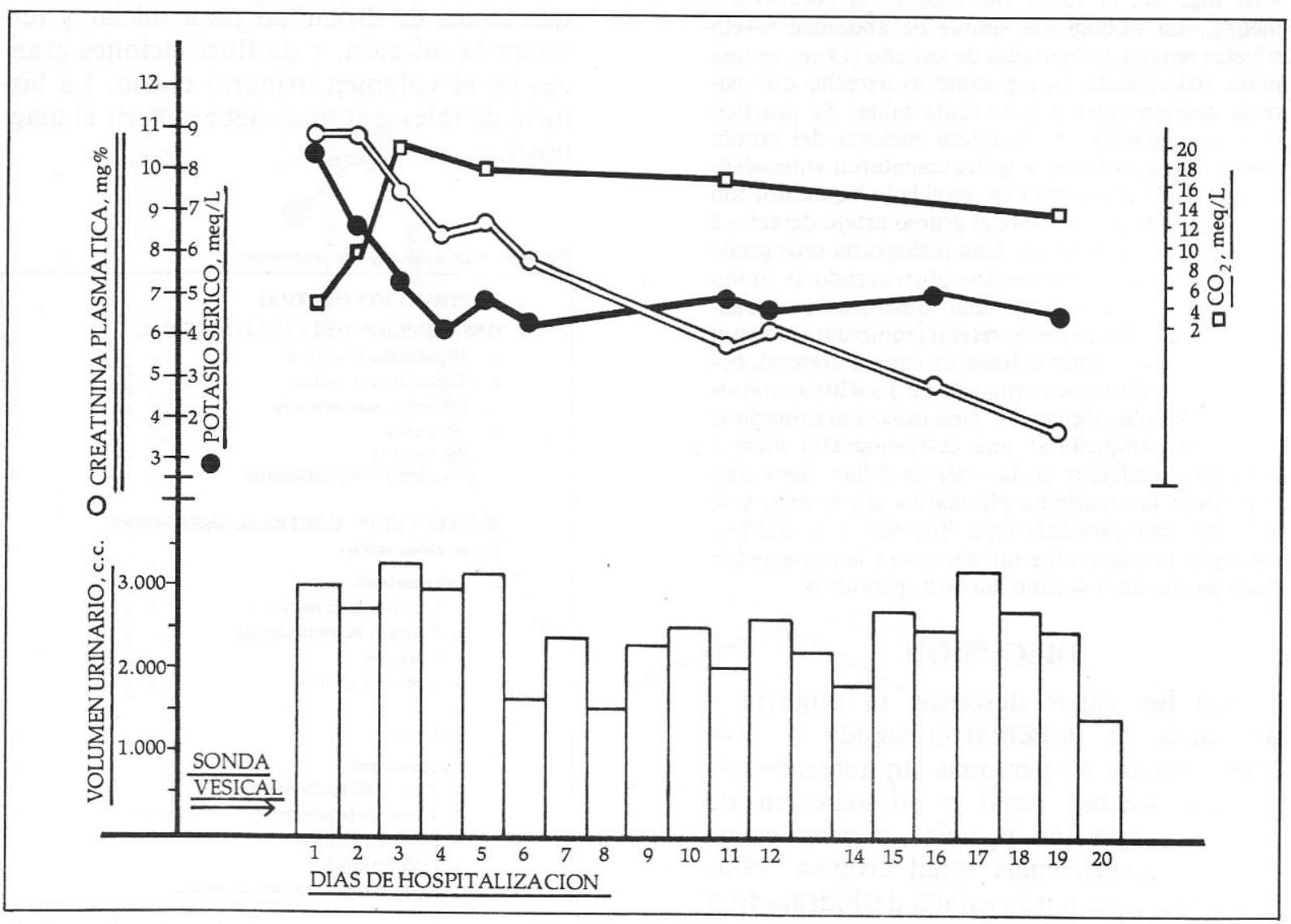

Gráfica 1- Caso No. 1 (GR). Curso clínico. 
la izquierda. Una radiografia mostró calcificaciones en la pelvis; las sombras renales no se visualizaron. Se colocó una sonda de Foley y se obtuvieron 3.000 c.c. de orina; durante la semana siguiente la diuresis fluctuó entre 3 y 4 litros diarios y las químicas sanguíneas se normalizaron. Un legrado-biopsia mostró displasia adenoquística del endometrio; la exploración quirúrgica reveló un mioma uterino de $30 \mathrm{~cm}$. de diámetro con centro necrótico, que se resecó con una histerectomía total. La paciente fue dada de alta en el octavo día postoperatorio con creatinina plasmática y electrolitos normales.

\section{Caso No. 4}

L.C., una mujer blanca de 49 años, fue enviada al Hospital con un cuadro de cólico abdominal localizado en flanco derecho e irradiado a la región lumbar y genital del mismo lado, acompañado de oliguria progresiva de 8 días de evolución; la paciente presentó deterioro rápido del estado general y de conciencia. El examen de ingreso mostró una mujer obesa, letárgica y desorientada; el corazón y los pulmones eran normales, el abdomen era doloroso, defendido, con una masa dolorosa de difícil delimitación en hipocondrio derecho. No había edemas. El hematocrito era $34 \%$, con leucograma normal; el nitrógeno ureico $65.3 \mathrm{mg}$. \%, la creatinina plasmática $9.64 \mathrm{mg}$. \%, el sodio $140 \mathrm{meq} / \mathrm{L}$, el potasio 5.5 $\mathrm{meq} / \mathrm{L}$. La radiografía simple de abdomen reveló siluetas renales aumentadas de tamaño $(13 \mathrm{~cm}$. $)$ y una masa redondeada en hipocondrio derecho que parecía corresponder a la vesícula biliar. Se practicó una hemodiálisis obteniéndose mejoría del estado mental de la paciente, y se documentaron antecedentes de un cólico similar pero en el lado izquierdo, con dolor e inflamación sobre el grueso artejo derecho 5 meses antes del ingreso. Una pielografía retrógrada mostró cálculos radiolúcidos obstruyendo la unión pieloureteral derecha y una obstrucción infranqueable en la unión pieloureteral izquierda; sólo en el lado derecho se pudo colocar un catéter ureteral, obteniéndose volúmenes urinarios de 3 a 4 litros diarios durante los días siguientes. Una infección urinaria se trató con Ampicilina; una colecistografia mostró numerosos cálculos en la vesícula biliar. Siete días más tarde la creatinina plasmática era normal y se practicó una pielolitotomía bilateral y colecistectomia; la evolución fue satisfactoria y la paciente fue dada de alta en el décimo día postoperatorio.

\section{DISCUSION}

En los casos descritos la oliguria y azotemia se presentaron aguda y progresivamente en personas sin antecedentes de enfermedad renal y en ausencia de anemia, depleción proteica $\mathrm{u}$ otros estigmas de insuficiencia renal crónica. Ninguno de los pacientes estaba deshidratado o hipovolémico, y aunque el paciente $\mathrm{N}^{\circ} 2$ presentaba signos de insuficiencia cardiaca congestiva, ésta parecía ser más la consecuencia que la causa del flujo urinario disminuido. Por otra parte, la oliguria fue progresiva, irregular y terminó frecuentemente en anuria completa; se imponía en cada caso la investigación de una posible obstrucción urinaria.

La causa más frecuente de oliguria aguda severa y anuria ( 0 a 50 c.c. en 24 horas) es la obstrucción del cuello vesical por una próstata hiperplásica en los varones (Tabla 3); de hecho, la otra causa de anuria completa es la necrosis cortical renal, que generalmente ocurre en situaciones clínicas bien diferentes $(6,7)$. La obstrucción uretral es más rara en el sexo femenino y cuando ocurre, como en nuestro caso $\mathrm{N}^{\circ} 3$, se debe generalmente al desarrollo de masas pélvicas. En ambos sexos, sin embargo, suelen encontrarse antecedentes de dificultad para iniciar y terminar la micción, y de fluctuaciones grandes en el volumen urinario diario. La historia de tales síntomas debe sugerir el diagnóstico.

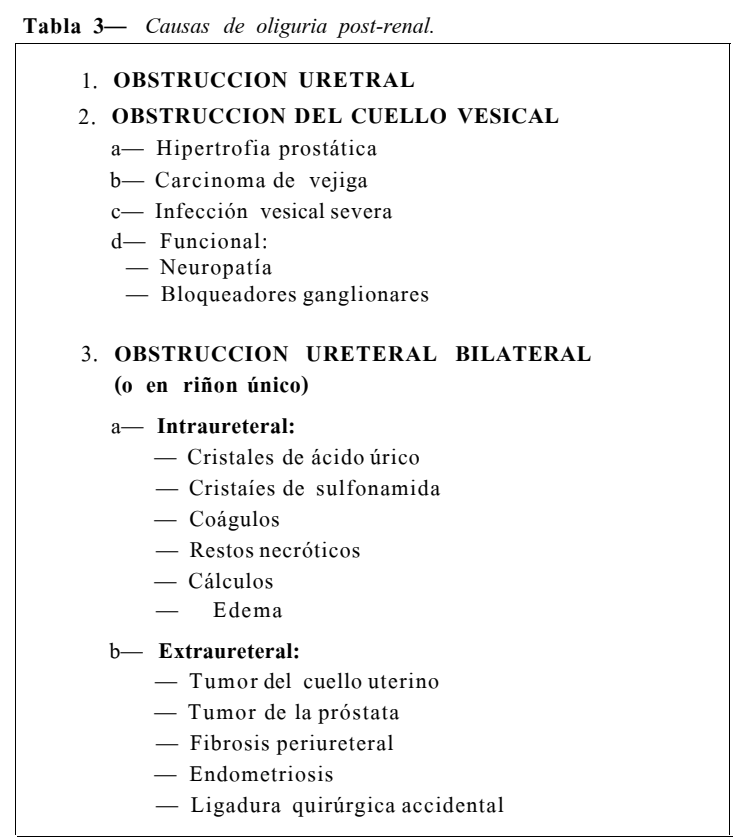


La obstrucción de las vías urinarias altas debe ser bilateral u ocurrir en riñón único para producir oliguria, y es entonces extremadamente rara; sin embargo, debe considerarse siempre en casos oscuros de oliguria, como se ilustra en este informe. Si la obstrucción se sospecha clínicamente, o por el hallazgo de sombras renales aumentadas de tamaño en la radiografía simple de abdomen, debe excluirse definitivamente por medio de una urografía excretora o, más frecuentemente, por una pielografía retrógrada; este último procedimiento está absolutamente indicado en casos de oliguria en riñón único. Cuando se dispone de recursos adecuados, la sonografía es un medio no invasivo y satisfactorio para identificar estructuras pielocaliciales dilatadas $(9,10)$.

El alivio de la obstrucción urinaria bilateral conduce generalmente a una

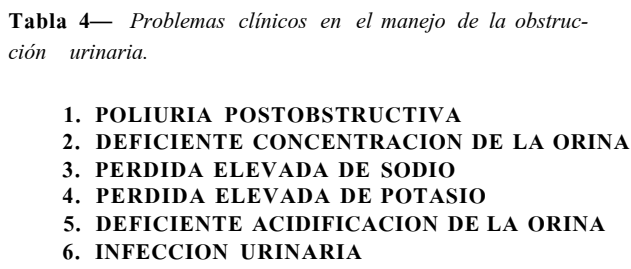

rápida mejoría de la oliguria y de la retención nitrogenada; sin embargo, presenta también problemas serios en el manejo clínico del paciente (Tabla 4). Frecuentemente se observa una marcada poliuria postobstructiva (11), una marcada pérdida de sodio y de potasio y una deficiencia variable en la acidificación de la orina (12); además, la filtración glomerular disminuye $\mathrm{y}$ en ocasiones no regresa a la normalidad, especialmente cuando la obstrucción ha sido prolongada (13). Las gráficas 1 y 2 , correspondientes a los dos primeros casos

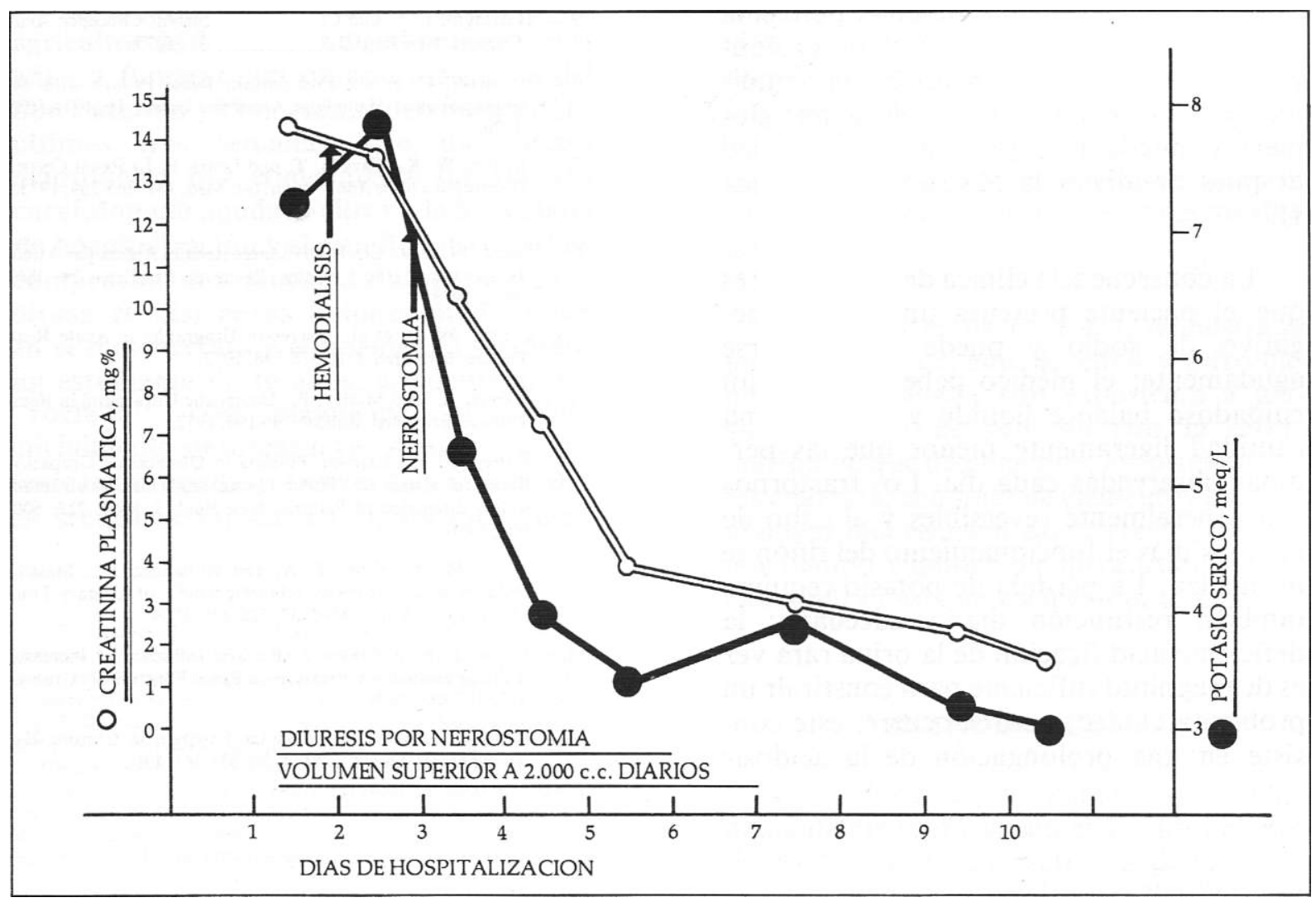

Gráfica 2-Caso No. 2 (BB). Curso clínico. 
de este informe, ilustran esas anormalidades.

Las causas de estos trastornos son varias e incluyen una diuresis osmótica por la retención nitrogenada, la brusca eliminación de todo el sodio y el agua acumulados mientras la obstrucción persiste, y una deficiencia específica en la reabsorción distal de sodio en el túbulo renal, dependiente al parecer de la presión aumentada de su contenido (14). Cuando el flujo urinario cesa por obstrucción urinaria bilateral, la presión hidrostática dentro del nefrón alcanza rápidamente valores de $80 \mathrm{a}$ $90 \mathrm{~mm} . \mathrm{Hg}$; en estas condiciones la filtración glomerular disminuye hasta cesar por completo. Estudios hemodinámicos (13) indican que los nefrones yuxtamedulares son más afectados que los corticales; si el agua y los solutos se reabsorben parcialmente y la presión hidrostática disminuye, la filtración glomerular puede restablecerse en forma intermitente, pero si la obstrucción permanece por tiempo prolongado, se producirá un daño parenquimatoso irreversible y la filtración glomerular puede no regresar a la normalidad después de aliviar la obstrucción (Gráfica 1).

La consecuencia clínica de lo anterior es que el paciente presenta un balance negativo de sodio y puede deshidratarse agudamente; el médico debe observar un cuidadoso balance líquido y restituir una cantidad ligeramente menor que las pérdidas observadas cada día. Los trastornos son generalmente reversibles y al cabo de algunos días el funcionamiento del riñon se normaliza. La pérdida de potasio requiere también restitución diaria adecuada; la deficiente acidificación de la orina rara vez es de magnitud suficiente para constituir un problema clínico, pero si ocurre, éste consiste en una prolongación de la acidosis metabólica que usualmente presenta el paciente obstruido y azotémico.

\section{SUMMARY}

Four cases of acute oliguria produced by obstruction of the urinary tract are described and the differential diagnosis with other causes of acute oliguria is discussed. An outline of the physiological disturbances produced in the obstructed kidney is also included and their clinical management discussed.

\section{BIBLIOGRAFIA}

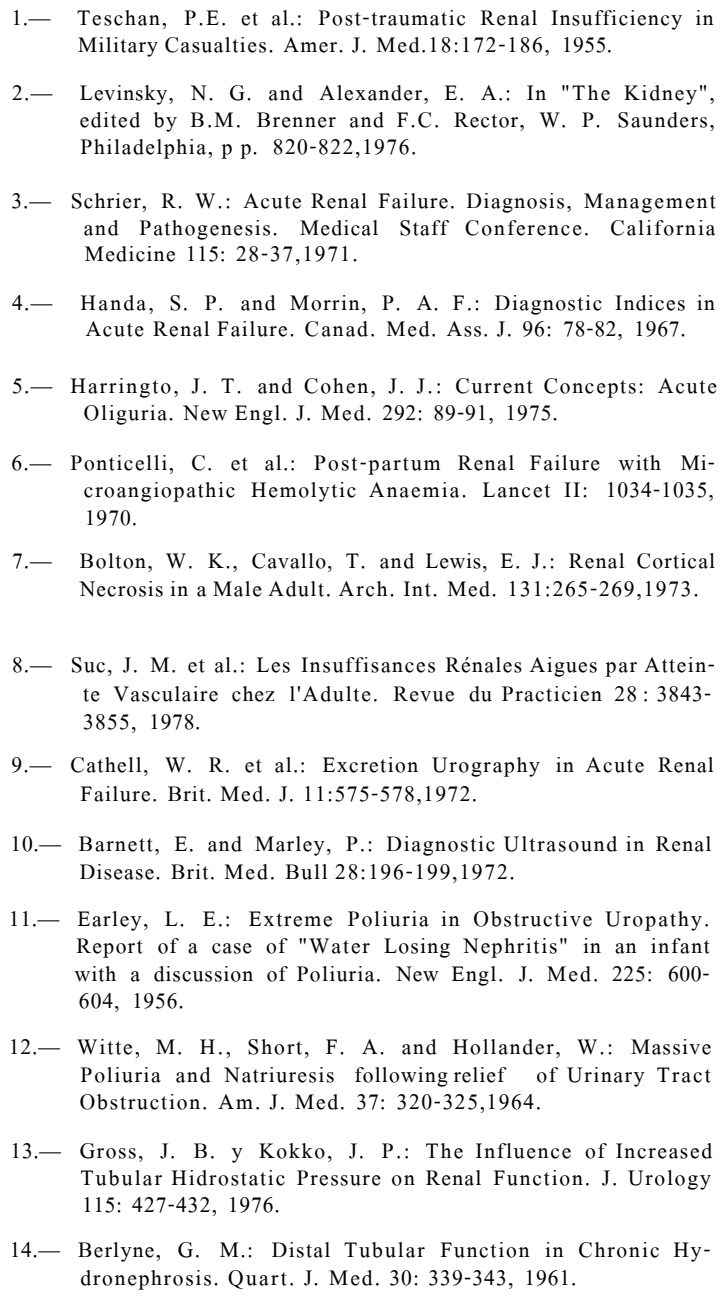

1.- Teschan, P.E. et al.: Post-traumatic Renal Insufficiency in Military Casualties. Amer. J. Med.18:172-186, 1955.

2.- Levinsky, N. G. and Alexander, E. A.: In "The Kidney", edited by B.M. Brenner and F.C. Rector, W. P. Saunders, Philadelphia, p p. 820-822,1976.

3.- Schrier, R. W.: Acute Renal Failure. Diagnosis, Management and Pathogenesis. Medical Staff Conference. California Medicine 115: 28-37,1971

4.- Handa, S. P. and Morrin, P. A. F.: Diagnostic Indices in Acute Renal Failure. Canad. Med. Ass. J. 96: 78-82, 1967.

5.- Harringto, J. T. and Cohen, J. J.: Current Concepts: Acute Oliguria. New Engl. J. Med. 292: 89-91, 1975.

6.- Ponticelli, C. et al.: Post-partum Renal Failure with Microangiopathic Hemolytic Anaemia. Lancet II: 1034-1035, 1970.

7.- Bolton, W. K., Cavallo, T. and Lewis, E. J.: Renal Cortical Necrosis in a Male Adult. Arch. Int. Med. 131:265-269,1973.

8.- Suc, J. M. et al.: Les Insuffisances Rénales Aigues par Atteinte Vasculaire chez l'Adulte. Revue du Practicien 28:38433855,1978 .

9.- Cathell, W. R. et al.: Excretion Urography in Acute Renal Failure. Brit. Med. J. 11:575-578,1972.

10.- Barnett, E. and Marley, P.: Diagnostic Ultrasound in Renal Disease. Brit. Med. Bull 28:196-199,1972.

11.- Earley, L. E.: Extreme Poliuria in Obstructive Uropathy. Report of a case of "Water Losing Nephritis" in an infant with a discussion of Poliuria. New Engl. J. Med. 225: 600$604,1956$.

12.- Witte, M. H., Short, F. A. and Hollander, W.: Massive Poliuria and Natriuresis following relief of Urinary Tract Obstruction. Am. J. Med. 37: 320-325,1964.

13.- Gross, J. B. y Kokko, J. P.: The Influence of Increased Tubular Hidrostatic Pressure on Renal Function. J. Urology 115: 427-432, 1976

14.- Berlyne, G. M.: Distal Tubular Function in Chronic Hydronephrosis. Quart. J. Med. 30: 339-343, 1961. 